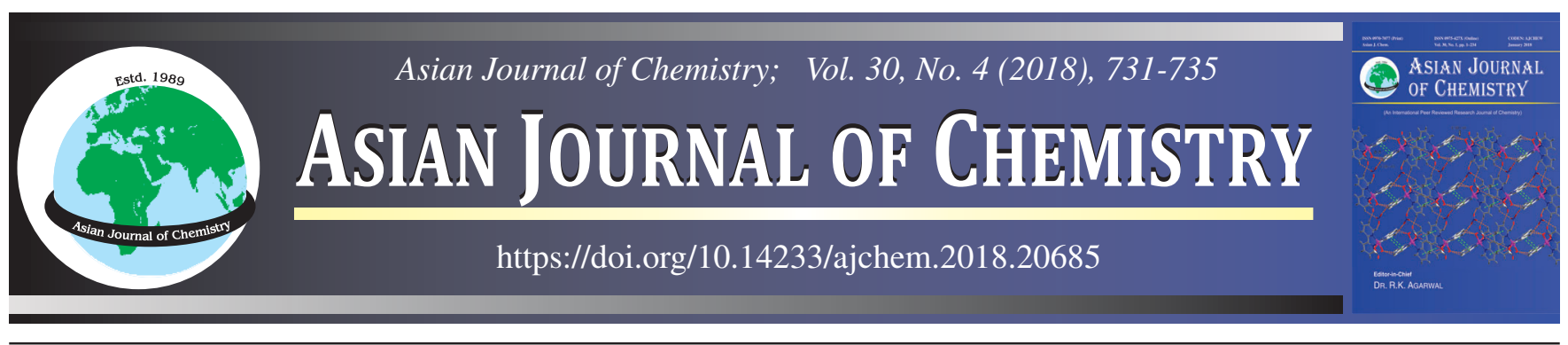

\title{
Measurements and Modeling of Excess Molar Enthalpy of Binary Mixtures of Oxygenate and Hydrocarbons
}

\author{
Suman Gahlyan ${ }^{1}$, Sweety Verma ${ }^{1}$, Manju Rani ${ }^{2}$ and Sanjeev Maken ${ }^{1, *}$
}

${ }^{1}$ Department of Chemistry, Deenbandhu Chhotu Ram University of Science and Technology, Murthal-131 039, India

${ }^{2}$ Department of Chemical Engineering, Deenbandhu Chhotu Ram University of Science and Technology, Murthal-131 039, India

*Corresponding author: E-mail: sanjeevmakin@gmail.com

Received: 3 April 2017;

Accepted: 15 June 2017;

Published online: 28 February 2018;

AJC-18773

| The thermodynamic properties of the binary mixtures having potential oxygenates, biodiesel, aromatic and aliphatic compounds are of considerable importance to chemical engineers in formulation of motor fuel. In order to study the interactions of oxygenate with fuel components, excess molar enthalpy for 2-propanol (1) + aromatic hydrocarbon (2) mixtures were measured with flow microcalorimeter at $303.15 \mathrm{~K}$. At equimole fraction, excess enthalpy values follow the order as: $n$-hexane > cyclohexane and 2-propanol > 1-propanol. The data have also been interpreted in terms of graph-theoretical approach and Prigogine-Flory-Patterson (PFP) theory and FloryTreszczanowicz-Benson association (FTB) model. It has been observed that while Prigogine-Flory-Patterson theory fails to predict the excess enthalpy $\left(\mathrm{H}_{m}^{\mathrm{E}}\right)$ values for these system in the composition range $\mathrm{x}_{1}>0.05$, agreement with experimental values is reasonably good in propanol lean region. Flory-Treszczanowicz-Benson $(\mathrm{FTB})$ association model and graphtheoretical approach predict the measured $\mathrm{H}_{\mathrm{m}}^{\mathrm{E}}$ quite well.

Keywords: Fuel oxygenate, 2-Propanol, Prigogine-Flory-Patterson theory, Flory-Treszczanowicz-Benson model.

ᄂ

\section{INTRODUCTION}

Due to price hikes, rapid depletion of fossil fuels and strict emission regulation, considerable concern has been raised over diesel-powered vehicles using alternative fuels. Bio-fuels for internal combustion engines as oxygenated compounds are also becoming important because of diminishing petroleum reserves and increasing air pollution [1-5]. The oxygenated compounds used worldwide as gasoline additives to reduce pollutants from vehicle exhaust gases [4]. Oxygenates are the compounds that contain oxygen such as alcohol, ethers, glycol ethers, methylal and carbonates or biomass products such as esters of vegetable oils [6]. Oxygenates have many advantages as they are octane enhancers, have significant anti-knock properties important for unleaded fuel, can be produced from renewable agricultural and raw materials instead of fossil sources and they reduce carbon monoxide pollution from vehicle exhaust [7-9].

Thus, the thermo-physical properties of oxygenate additive with aromatic hydrocarbons as well as with aliphatic hydrocarbon would be of great significance in process engineering design and in formulating motor gasoline and diesel [10]. As the excess properties are measure of departure from ideality, excess enthalpy of mixtures having oxygenate would also be of great interest to chemists in getting information about the energetics of intermolecular interactions in these binary mixtures $[11,12]$. These considerations led us to carry out a systemic study of the thermodynamics of binary mixtures of alkanol with alkane or aromatic hydrocarbon. In continuation of earlier work, this paper reports the excess molar enthalpy $\left(\mathrm{H}_{\mathrm{m}}{ }^{\mathrm{E}}\right)$ of 2-propanol + aromatic hydrocarbons mixtures at 303.15 K [13-15].

\section{EXPERIMENTAL}

Cyclohexane, $n$-hexane and isomer of propanol (Merck) were purified by the standard procedures $[16,17]$. The purities of purified sample were checked by measuring their densities and refractive indices using specially designed densimeter and Abbe refractometer (OSAW, India) in the manner described earlier [13-15]. The accuracy in the measurement of density and refractive indices were $\pm 0.05 \mathrm{~kg} \mathrm{~m}^{-3}$ and \pm 0.001 unit. Measured value of densities and refractive indices of the pure compounds agree well with their respective literature values and shown in earlier work [13-15]. The purified samples were also analyzed by gas chromatography for their purity and found to have better than $99.6 \mathrm{wt}$. \%

The excess molar enthalpy of mixing $\left(\mathrm{H}_{\mathrm{m}}^{\mathrm{E}}\right)$ values at 303.15 $\pm 0.01 \mathrm{~K}$ for the various $(1+2)$ mixtures were measured using flow microcalorimeter (LKB-2107, Bromma, Sweden) in the 
manner described by Monk et al. [18] and recorded in Table-1 . Details and the operating procedure of the apparatus have been described elsewhere $[19,20]$. The accuracy of the measurement was checked by measuring the enthalpy of mixing for benzene + carbon tetrachloride mixtures at $298.15 \mathrm{~K}$ and these agreed with the literature values within $\pm 2 \mathrm{~J} \mathrm{~mol}^{-1}[21]$.

\section{RESULTS AND DISCUSSION}

The measured $\mathrm{H}_{\mathrm{m}}^{\mathrm{E}}$ data were fitted to the following Redlich and Kister equation [22]:

$$
\mathrm{H}_{\mathrm{m}}^{\mathrm{E}}\left(\mathrm{J} \mathrm{mol}^{-1}\right)=\mathrm{x}_{1}\left(1-\mathrm{x}_{1}\right)\left(\sum_{\mathrm{n}=0}^{3} \mathrm{H}^{(\mathrm{n})}\left(1-2 \mathrm{x}_{1}\right)^{\mathrm{n}}\right)
$$

where $\mathrm{H}^{(\mathrm{n})}$ are the adjustable parameters and $\mathrm{x}_{1}$ is the mole fractions of isomer of propanol (1) in binary $(1+2)$ mixture. These parameters were evaluated by fitting $\mathrm{H}_{\mathrm{m}}^{\mathrm{E}}$ data to eqn. 1 by least squares method and recorded in Table- 2 along with the standard deviations of $\mathrm{H}_{\mathrm{m}}^{\mathrm{E}},\left(\sigma\left(\mathrm{H}_{\mathrm{m}}^{\mathrm{E}}\right)\right)$ [15]. The measured and smoothened values from eqn. 1 are shown in Fig. 1 as a function of mole fraction of isomer of propanol (1).

The $\mathrm{H}_{\mathrm{m}}^{\mathrm{E}}$ versus $\mathrm{x}_{1}$ plots for isomer of propanol (1) + cyclohexane or $n$-hexane systems are skewed with positivel $\mathrm{H}_{\mathrm{m}}^{\mathrm{E}}$ values over the whole composition range (Fig. 1).

At equimolar composition, excess enthalpy values for binary mixtures follow the order: $n$-hexane $>$ cyclohexane and 2propanol > 1-propanol. The positive contribution to $\mathrm{H}_{\mathrm{m}}^{\mathrm{E}}$ values arises from the breaking of self-associated isomer of propanol and dipole-dipole interactions between monomers and dimers of isomer of propanol and also from the disruption in favourable orientation order of hydrocarbons. Next we analyzed

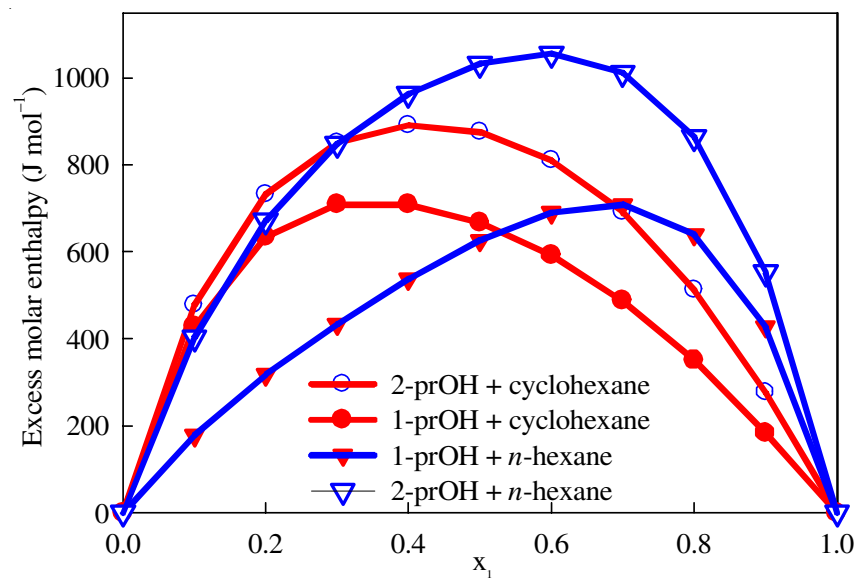

Fig. 1. Excess molar enthalpy $\left(\mathrm{H}_{\mathrm{m}}^{\mathrm{E}}\right)$ of isomer of propanol $(1)+$ cyclohexane or $n$-hexane (2) mixture as a function of mole fraction of propanol $\left(\mathrm{x}_{1}\right)$ at $303.15 \mathrm{~K}$; symbols represent experimental values and lines represent values calculated from eqn. 1

the present $\mathrm{H}_{\mathrm{m}}^{\mathrm{E}}$ values in terms of graph theoretical approach $[23,24]$, Prigogine-Flory-Patterson theory [25-30] and FloryTreszczanowicz-Benson association model [31].

Graph theoretical approach: In order to understand the energetics of the various interactions present in isomer of propanol (1) + cyclohexane or $n$-hexane mixtures, it is assumed that the process of mixtures formation requires:

(a) a mixing of $\left(1_{n}\right)$ with (2) to establish $\left(1_{n}\right)-(2)$ contacts, (b) these $\left(1_{\mathrm{n}}\right)-(2)$ contacts would then cause rupture of intermolecular association in isomer of propanol to yield monomers and (c) the monomers of 2-propanol then interact with aromatic hydrocarbon to give (1)-(2) molecular entity.

TABLE-1

MEASURED EXCESS MOLAR ENTHALPIES, $\mathrm{H}^{\mathrm{E}}\left(\mathrm{J} \mathrm{mol}^{-1}\right)$ FOR BINARY MIXTURE OF ISOMER OF PROPANOL (1) + HYDROCARBONS (2) AS FUNCTIONS OF MOLE FRACTION OF PROPANOL AT 303.15 K

\begin{tabular}{|c|c|c|c|c|c|c|c|}
\hline \multicolumn{4}{|c|}{ 1-Propanol (1) + hydrocarbon (2) } & \multicolumn{4}{|c|}{ 2-Propanol (1) + hydrocarbon (2) } \\
\hline \multicolumn{2}{|c|}{ Cyclohexane } & \multicolumn{2}{|c|}{$n$-Hexane } & \multicolumn{2}{|c|}{ Cyclohexane } & \multicolumn{2}{|c|}{$n$-Hexane } \\
\hline $\mathrm{x}_{1}$ & $\mathrm{H}^{\mathrm{E}}$ & $\mathrm{x}_{1}$ & $\mathrm{H}^{\mathrm{E}}$ & $\mathrm{x}_{1}$ & $\mathrm{H}^{\mathrm{E}}$ & $\mathrm{x}_{1}$ & $\mathrm{H}^{\mathrm{E}}$ \\
\hline 0.0695 & 325 & 0.0331 & 620 & 0.0348 & 202 & 0.0288 & 134 \\
\hline 0.1234 & 493 & 0.1021 & 181 & 0.0766 & 390 & 0.0696 & 294 \\
\hline 0.1893 & 617 & 0.1752 & 282 & 0.1159 & 532 & 0.1159 & 452 \\
\hline 0.2348 & 675 & 0.2338 & 362 & 0.1834 & 703 & 0.1734 & 611 \\
\hline 0.2895 & 707 & 0.2971 & 433 & 0.2474 & 804 & 0.2374 & 748 \\
\hline 0.3560 & 716 & 0.3569 & 494 & 0.3086 & 856 & 0.3186 & 873 \\
\hline 0.4253 & 697 & 0.4342 & 565 & 0.3709 & 882 & 0.3809 & 943 \\
\hline 0.4817 & 674 & 0.4944 & 618 & 0.4561 & 886 & 0.4361 & 993 \\
\hline 0.5301 & 648 & 0.5651 & 673 & 0.5159 & 869 & 0.5059 & 1038 \\
\hline 0.5880 & 604 & 0.6039 & 694 & 0.5834 & 822 & 0.5634 & 1052 \\
\hline 0.6353 & 560 & 0.6670 & 710 & 0.6362 & 776 & 0.6162 & 1056 \\
\hline 0.6901 & 497 & 0.7138 & 702 & 0.6904 & 703 & 0.6704 & 1031 \\
\hline 0.7734 & 395 & 0.7704 & 676 & 0.7502 & 612 & 0.7202 & 994 \\
\hline 0.8315 & 302 & 0.8531 & 551 & 0.8036 & 506 & 0.8036 & 856 \\
\hline 0.8902 & 199 & 0.8898 & 457 & 0.8666 & 361 & 0.8466 & 744 \\
\hline 0.9325 & 120 & 0.9509 & 239 & 0.9390 & 169 & 0.9490 & 316 \\
\hline
\end{tabular}

TABLE-2

ADJUSTABLE PARAMETERS OF REDLICH KISTER EQUATION AND STANDARD DEVIATION $\left(\sigma, \mathrm{J}_{\text {mol }}^{-1}\right)$ AT $303.15 \mathrm{~K}^{-1}$

\begin{tabular}{lccccc}
\multicolumn{1}{c}{ System } & $\mathrm{H}^{(1)}$ & $\mathrm{H}^{(2)}$ & $\mathrm{H}^{(3)}$ & $\mathrm{H}^{(4)}$ & \\
\hline 1-Propanol (1) + Cyclohexane (2) & 2664 & 1174 & 1151 & 843 & 3 \\
1-Propanol (1) + n-Hexane (2) & 2503 & 1592 & 1349 & 222 & 3 \\
2-Propanol (1) + Cyclohexane (2) & 3501 & 802 & 1081 & 932 & 3 \\
2-Propanol (1) + n-Hexane (2) & 4133 & -957 & 1837 & -136 & 2 \\
\hline
\end{tabular}


The total enthalpy change due to processes (a), (b) and (c) [13-15] is given as:

$$
\mathrm{H}_{\mathrm{m}}^{\mathrm{E}}=\left(\frac{\mathrm{x}_{1} \mathrm{x}_{2}\left({ }^{3} \xi_{1} / \rho^{3} \xi_{2}\right)}{\mathrm{x}_{1}+\mathrm{x}_{2}\left({ }^{3} \xi_{1} / \beta^{3} \xi_{2}\right)}\right)\left[\left(1+\mathrm{x}_{2}\right) \chi_{12}^{\dagger}+\mathrm{x}_{1} \chi_{11}\right]
$$

Calculation of $\mathrm{H}_{\mathrm{m}}^{\mathrm{E}}$ from eqn. 2 requires the knowledge of connectivity parameter of third degree of ith component $\left({ }^{3} \xi_{\mathrm{i}}\right)$ (Table-3), two unknown interaction parameters $\chi_{12}^{\dagger}$ and $\chi_{11}$. These parameters were calculated using $\mathrm{H}_{\mathrm{m}}^{\mathrm{E}}$ values at two compositions $\left(\mathrm{x}_{1}=0.4\right.$ and 0.5$)$ for various binary mixtures and tabulated in Table- 4 . These parameters $\chi_{12}^{\dagger}$ and $\chi_{11}$ were subsequently used to evaluate $\mathrm{H}_{\mathrm{m}}^{\mathrm{E}}$ at other mole fractions (Table-5). The calculated $\mathrm{H}_{\mathrm{m}}^{\mathrm{E}}$ values found to agree well with their corresponding experimental values (Fig. 2). This lends additional support to the assumptions made in the derivation of eqn. 2.
Prigogine-Flory-Patterson (PFP) theory: According to Prigogine-Flory-Patterson theory [28], excess molar enthalpy $\left(\mathrm{H}_{\mathrm{m}}^{\mathrm{E}}\right)$ is expressed as sum of two contributions (i) interactional contribution and (ii) free volume contribution. Thus, $\mathrm{H}_{\mathrm{m}}^{\mathrm{E}}$ be expressed as:

$$
\begin{gathered}
\mathrm{H}_{\mathrm{m}}^{\mathrm{E}}=\mathrm{H}_{\text {Inter }}^{\mathrm{E}}+\mathrm{H}_{\text {free vol }}^{\mathrm{E}} \\
\mathrm{H}_{\mathrm{m}}^{\mathrm{E}}=\left(\mathrm{x}_{1} \mathrm{U}_{1}^{*}+\mathrm{x}_{2} \mathrm{U}_{2}^{*}\right)\left[-\tilde{\mathrm{U}}(\tilde{\mathrm{T}})+\tilde{\mathrm{T}} \tilde{\mathrm{C}}_{\mathrm{p}}(\tilde{\mathrm{T}})\right] \chi_{12} \psi_{1} \theta_{2} / \mathrm{P}_{1}^{*}+ \\
\left(\mathrm{x}_{1} \mathrm{U}_{1}^{*}+\mathrm{x}_{2} \mathrm{U}_{2}^{*}\right) \tilde{\mathrm{C}}_{\mathrm{p}}(\tilde{\mathrm{T}})\left(\psi_{1} \tilde{\mathrm{T}}_{1}+\psi_{2} \tilde{\mathrm{T}}_{2}-\tilde{\mathrm{T}}\right)
\end{gathered}
$$

where all the terms have usual meaning $[25,26]$. The Flory interaction parameters $\chi_{12}$ in eqn. 4 were calculated employing equimolar experimental $\mathrm{H}_{\mathrm{m}}^{\mathrm{E}}$ values, which were subsequently used to calculate $\mathrm{H}_{\mathrm{m}}^{\mathrm{E}}$ at other mole fraction $\left(\mathrm{x}_{1}\right)$ and compared with experimental values in Fig. 2 and Table-5. The calculated values of interactional contribution and free volume contribution

TABLE-3

CONNECTIVITY PARAMETERS OF THIRD ORDER ${ }^{3} \xi_{\mathrm{i}}$, MOLAR VOLUME (V), ISOBARIC EXPANSIVITY $(\alpha)$, ISOTHERMAL COMPRESSIBILITY $\left(\kappa_{\mathrm{T}}\right)$, CHARACTERISTIC PRESSURE $\left(\mathrm{P}^{*}\right)$, CHARACTERISTIC MOLAR VOLUME (V*) AND CHARACTERISTIC TEMPERATURE (T*) OBTAINED FROM FLORY THEORY FOR THE PURE LIQUIDS AT $303.15 \mathrm{~K}$

\begin{tabular}{lccccccc}
\hline \multicolumn{1}{c}{ Compound } & ${ }^{3} \xi_{\mathrm{i}}$ & $\mathrm{V}\left(\mathrm{cm}^{3} \mathrm{~mol}^{-1}\right)$ & $10^{3} \alpha\left(\mathrm{K}^{-1}\right)$ & $10^{6} \kappa_{\mathrm{T}}\left(\mathrm{cm}^{3} \mathrm{~J}^{-1}\right)$ & $\mathrm{P} *\left(\mathrm{~J} \mathrm{~cm}^{-3}\right)$ & $\mathrm{V}^{*}\left(\mathrm{~cm}^{3} \mathrm{~mol}^{-1}\right)$ & $\mathrm{T}^{*}(\mathrm{~K})$ \\
\hline 1-Propanol & 0.4082 & 75.503 & 1.0344 & 679.79 & 730.272 & 60.010 & 5175 \\
2-Propanol & 0.4710 & 77.359 & 1.1437 & 828.66 & 730.272 & 60.010 \\
Cyclohexane & 1.5000 & 109.401 & 1.2320 & 1237.00 & 508.147 & 84.329 & 4732 \\
$n$-Hexane & 0.9500 & 131.610 & 1.4040 & 780.90 & 422.277 & 99.011 & 4452 \\
\hline
\end{tabular}

TABLE-4

VALUES OF THE VARIOUS PFP CONTRIBUTIONS TO EXCESS ENTHALPY $\left(\mathrm{J} \mathrm{mol}^{-1}\right)$, PFP INTERACTION PARAMETER $\left(\chi_{12}^{*}\right)$, FTB ASSOCIATION MODEL PARAMETERS K ${ }^{\phi}$ AND $\chi_{12}$ AND GRAPH THEORY INTERACTION PARAMETER $\xi_{12}^{\dagger}$ OR $\chi_{11}$ AT $303.15 \mathrm{~K}$

\begin{tabular}{lccccccc}
\hline \multicolumn{1}{c}{ System } & $\mathrm{H}_{\text {Inter }}^{\mathrm{E}}$ & $\mathrm{H}_{\text {Free vol }}^{\mathrm{E}}$ & $\chi_{12}^{*}$ & $\mathrm{~K}^{\phi}$ & $\chi_{12}$ & $\chi_{12}^{\dagger}$ \\
\hline 1-Propanol (1) + cyclohexane (2) & 681 & -15.05 & 38.80 & 234.4 & 11.34 & 5623 & 1205 \\
1-Propanol (1) + n-hexane (2) & 674 & -47.91 & 36.38 & 234.4 & -2.302 & 1312 \\
2-Propanol (1) + cyclohexane (2) & 882 & -2.76 & 49.83 & 232.9 & 28.68 & 5489 & 3657 \\
2-Propanol (1) + n-hexane (2) & 1055 & -21.79 & 56.42 & 232.9 & 31.63 & 3625 \\
\hline
\end{tabular}

TABLE-5

\begin{tabular}{|c|c|c|c|c|c|c|c|c|}
\hline \multirow{2}{*}{$\mathrm{x}_{1}$} & Exptl. & FTB & PFP & Graph & Exptl. & FTB & PFP & Graph \\
\hline & \multicolumn{4}{|c|}{ 1-Propanol (1) + cyclohexane (2) } & \multicolumn{4}{|c|}{ 2-Propanol (1) + cyclohexane (2) } \\
\hline 0.1 & 429 & 468 & 220 & 408 & 478 & 536 & 290 & 433 \\
\hline 0.2 & 634 & 597 & 400 & 611 & 732 & 722 & 526 & 693 \\
\hline 0.3 & 708 & 663 & 536 & 697 & 851 & 825 & 705 & 836 \\
\hline 0.4 & 708 & 683 & 625 & 708 & 891 & 880 & 822 & 891 \\
\hline 0.5 & 666 & 666 & 666 & 666 & 875 & 875 & 875 & 875 \\
\hline 0.6 & 592 & 606 & 653 & 585 & 810 & 824 & 859 & 799 \\
\hline 0.7 & 488 & 514 & 584 & 473 & 692 & 707 & 768 & 669 \\
\hline 0.8 & 351 & 388 & 456 & 336 & 513 & 528 & 599 & 490 \\
\hline \multirow[t]{2}{*}{0.9} & 183 & 221 & 263 & 177 & 277 & 287 & 345 & 267 \\
\hline & \multicolumn{4}{|c|}{ 1-Propanol (1) $+n$-hexane (2) } & \multicolumn{4}{|c|}{ 2-Propanol (1) $+n$-hexane (2) } \\
\hline 0.1 & 178 & 485 & 199 & 137 & 403 & 612 & 330 & 334 \\
\hline 0.2 & 318 & 602 & 364 & 280 & 671 & 843 & 604 & 608 \\
\hline 0.3 & 434 & 647 & 493 & 417 & 848 & 970 & 816 & 820 \\
\hline 0.4 & 537 & 648 & 581 & 537 & 963 & 1028 & 961 & 963 \\
\hline 0.5 & 626 & 626 & 626 & 626 & 1033 & 1033 & 1033 & 1033 \\
\hline 0.6 & 690 & 571 & 622 & 669 & 1056 & 980 & 1024 & 1023 \\
\hline 0.7 & 708 & 475 & 564 & 649 & 1012 & 848 & 927 & 925 \\
\hline 0.8 & 639 & 347 & 445 & 546 & 864 & 647 & 731 & 730 \\
\hline 0.9 & 428 & 195 & 260 & 338 & 553 & 370 & 426 & 426 \\
\hline
\end{tabular}

COMPARISON OF EXCESS MOLAR ENTHALPY $\left(\mathrm{H}^{\mathrm{E}}, \mathrm{J}^{\mathrm{mol}}{ }^{-1}\right)$ VALUES CALCULATED FROM GRAPH THEORY, PFP THEORY AND FTB MODEL WITH THEIR CORRESPONDING EXPERIMENTAL VALUES FOR THE VARIOUS BINARY MIXTURES AS FUNCTION OF MOLE FRACTION OF PROPANOL, $\mathrm{x}_{1}$, AT $303.15 \mathrm{~K}$ 


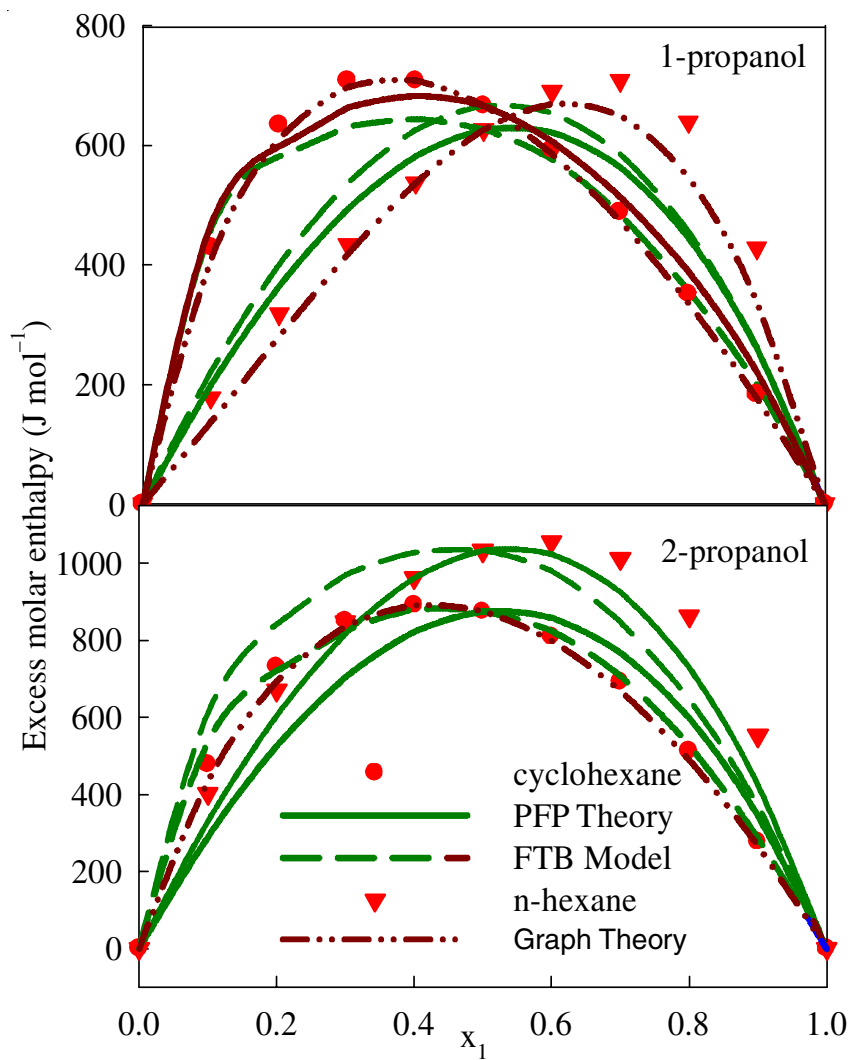

Fig. 2. Excess molar enthalpy $\left(\mathrm{H}_{\mathrm{m}}^{\mathrm{E}}\right)$ of isomer of propanol $(1)+$ cyclohexane or $n$-hexane (2) as a function of mole fraction of propanol $\left(\mathrm{x}_{1}\right)$ at $303.15 \mathrm{~K}$

to the value of $\mathrm{H}_{\mathrm{m}}^{\mathrm{E}}$ for equimolar composition and Flory interaction parameters are recorded in Table-3. It has been observed from Fig. 2 that PFP theory fails to predict $\mathrm{H}_{\mathrm{m}}^{\mathrm{E}}$ values for propanol (1) $+n$-hexane (2) systems systems in the composition range $\mathrm{x}_{1}>0.5$, agreement with experimental values is reasonably good in propanol lean region.

\section{Flory-Treszczanowicz-Benson (FTB) model [31]}

This model expresses $\mathrm{H}_{\mathrm{m}}^{\mathrm{E}}$ as:

$$
\mathrm{H}_{\mathrm{m}}^{\mathrm{E}}=\mathrm{H}_{\mathrm{Chem}}^{\mathrm{E}}+\mathrm{H}_{\text {Phys }}^{\mathrm{E}}
$$

where

$$
\mathrm{H}_{\mathrm{Chem}}^{\mathrm{E}}=\Delta \mathrm{h}_{\mathrm{H}}^{\mathrm{o}} \mathrm{x}_{1} \mathrm{~h}\left(\mathrm{~K}^{\phi}, \phi_{1}\right)
$$

In eqn. $6, \mathrm{~K}^{\phi}$ is an association constant expressed in volume fraction and given as:

$$
\ln \mathrm{K}^{\phi}=1+\ln \left(\mathrm{K}_{\mathrm{H}} / \mathrm{r}_{1}\right)
$$

where $\mathrm{K}_{\mathrm{H}}=\exp \left(-\frac{\left(\Delta \mathrm{h}_{\mathrm{H}}^{\mathrm{o}}-\mathrm{T} \Delta \mathrm{S}_{\mathrm{H}}^{\mathrm{o}}\right)}{\mathrm{RT}}\right)$ and $\mathrm{r}_{1}=\left(\mathrm{V}_{1}^{*} / 17.12 \mathrm{~cm}^{3}\right.$ $\left.\mathrm{mol}^{-1}\right)$. [25,26]:

The physical contribution $\mathrm{H}_{\text {Phys }}^{\mathrm{E}}$ is given by Flory theory

$$
\mathrm{H}_{\text {Phys }}^{\mathrm{E}}=\mathrm{H}_{\mathrm{F}}^{\mathrm{E}}=\mathrm{x}_{1} \theta_{2}\left(\frac{\mathrm{V}_{1}^{*}}{\tilde{\mathrm{V}}}\right) \chi_{12}+\sum_{\mathrm{i}=1}^{2}\left[\mathrm{x}_{\mathrm{i}} \mathrm{P}_{\mathrm{i}}^{*} \mathrm{~V}_{\mathrm{i}}^{*}\left(\tilde{\mathrm{V}}_{\mathrm{i}}^{-1}-\tilde{\mathrm{V}}^{-1}\right)\right](8)
$$

In these equations all the terms have their usual meaning $[25,26,31]$. The values of association parameters $\Delta \mathrm{v}_{\mathrm{H}}^{\circ}, \Delta \mathrm{h}_{\mathrm{H}}^{\circ}$ and $\Delta \mathrm{s}_{\mathrm{H}}^{\circ}$ used to calculate $\mathrm{H}_{\text {Chem }}^{\mathrm{E}}$ from eqn. 6 are taken as $-10 \mathrm{~cm}^{3}$ $\mathrm{mol}^{-1},-24400 \mathrm{~J} \mathrm{~mol}^{-1}$ and $-33 \mathrm{~J} \mathrm{~K}^{-1} \mathrm{~mol}^{-1}$, respectively [31]. Method of calculation of $\chi_{12}$ using equimolar experimental excess enthalpy was reported earlier $[32,33]$ and their values were calculated for the present systems using equimolar $\mathrm{V}_{\mathrm{m}}^{\mathrm{E}}$ data [14] and given in Table-4. The parameters required for the calculation of $\mathrm{H}_{\text {Phys }}^{\mathrm{E}}$ form Flory theory were also given in Table-3. A good agreement of calculated and experimental $\mathrm{H}_{\mathrm{m}}^{\mathrm{E}}$ values was observed in Fig. 2 and Table-5.

\section{Conclusion}

Excess molar enthalpy $\left(\mathrm{H}_{\mathrm{m}}^{\mathrm{E}}\right)$ for isomer of propanol + cyclohexane or $n$-hexane mixtures were measured by flow microcalorimeter at $303.15 \mathrm{~K}$. In order to study the interactions of oxygenate with fuel components, excess molar enthalpy for isomer of propanol (1) + cyclohexane or $n$-hexane (2) mixtures were measured with flow microcalorimeter at $303.15 \mathrm{~K}$. At equimolar fraction, excess enthalpy values for binary mixtures follow the order: $n$-hexane $>$ cyclohexane and 2-propanol > 1-propanol. The data have also been interpreted in terms of graph-theoretical approach, Prigogine-Flory-Patterson (PFP) theory and Flory-Treszczanowicz-Benson association (FTB) model. It has been observed that PFP theory fails to predict the $\mathrm{H}_{\mathrm{m}}^{\mathrm{E}}$ values for propanol (1) $+n$-hexane (2) systems systems in the composition range $\mathrm{x}_{1}>0.5$, agreement with experimental values is reasonably good in propanol lean region, However, Flory-Treszczanowicz-Benson (FTB) association model and graph-theoretical approach predict the measured $\mathrm{H}_{\mathrm{m}}^{\mathrm{E}}$ values quite well.

\section{ACKNOWLEDGEMENTS}

One of the authors Suman Gahlyan thanks the University Grant Commission, New Delhi for the award of Senior Research Fellowship.

\section{REFERENCES}

1. F. Aguilar, F.E.M. Alaoui, J.J. Segovia, M.A. Villamañán and E.A. Montero, Fluid Phase Equilib., 315, 1 (2012);

https://doi.org/10.1016/j.fluid.2011.11.005.

2. F.E.M. Alaoui, E.A. Montero, J.-P. Bazile, F. Aguilar and C. Boned, J. Chem. Thermodyn., 43, 1768 (2011); https://doi.org/10.1016/j.jct.2011.06.006

3. F. Ancillotti and V. Fattore, Fuel Process. Technol., 57, 163 (1998); https://doi.org/10.1016/S0378-3820(98)00081-2.

4. P. McKendry, Bioresour. Technol., 83, 37 (2002); https://doi.org/10.1016/S0960-8524(01)00118-3.

5. S. Zhang, Y. Yan, T. Li and Z. Ren, Bioresour. Technol., 96, 545 (2005); https://doi.org/10.1016/j.biortech.2004.06.015.

6. A. Murcak, C. Hapimoglu, I. Çevik, M. Karabektas and G. Ergen, Fuel, 109, 582 (2013); https://doi.org/10.1016/j.fuel.2013.03.014.

7. E.W. de Menezes, R. Cataluña, D. Samios and R. Silva, Fuel, 85, 2567 (2006);

https://doi.org/10.1016/j.fuel.2006.04.014.

8. I.-C. Hwang, S.-J. Park and S.-J. In, J. Ind. Eng. Chem., 20, 3292 (2014); https://doi.org/10.1016/j.jiec.2013.12.010.

9. M. Karabektas, G. Ergen and M. Hosoz, Fuel, 115, 855 (2014); https://doi.org/10.1016/j.fuel.2012.12.062.

10. Q.N. Chan, Y. Bao and S. Kook, Fuel, 130, 228 (2014); https://doi.org/10.1016/j.fuel.2014.04.015.

11. A. Mejía, H. Segura and M. Cartes, Fuel, 116, 183 (2014); https://doi.org/10.1016/j.fuel.2013.07.107.

12. R. Sharma, R.C. Thakur and B. Saini, Asian J. Chem., 28, 2331 (2016); https://doi.org/10.14233/ajchem.2016.20010. 
13. S. Gahlyan, M. Rani, S. Maken, H. Kwon, K. Tak and I. Moon, J. Ind. Eng. Chem., 23, 299 (2015); https://doi.org/10.1016/j.jiec.2014.08.032.

14. S. Gahlyan, M. Rani and S. Maken, J. Mol. Liq., 199, 42 (2014); https://doi.org/10.1016/j.molliq.2014.08.011.

15. S. Gahlyan, M. Rani, I. Lee, I. Moon and S. Maken, Korean J. Chem. Eng., 32, 168 (2015); https://doi.org/10.1007/s11814-014-0200-6.

16. J.A. Riddick, W.B. Bunger and T.K. Sakano, Organic Solvents. Physical Properties and Methods of Purification, Wiley, New York, edn 4 (1986).

17. A.I. Vogel, A Text Book of Practical Organic Chemistry, ELBS Longman, London, edn 4 (1978).

18. P. Monk, I. Wadsö, P. Karvonen, A.I. Virtanen and J. Paasivirta, Acta Chem. Scand., 22, 1842 (1968); https://doi.org/10.3891/acta.chem.scand.22-1842.

19. D.S. Adcock and M.L. McGlashan, Proc. R. Soc. Lond. A Math. Phys. Sci., 226, 266 (1954); https://doi.org/10.1098/rspa.1954.0253.

20. A. Maken and S. Maken, J. Ind. Eng. Chem., 18, 1013 (2012); https://doi.org/10.1016/i.jiec.2011.11.139.

21. R.H. Stokes, K.N. Marsh and R.P. Tomlins, J. Chem. Thermodyn., 1, 211 (1969); https://doi.org/10.1016/0021-9614(69)90059-7.

22. O. Redlich and A.T. Kister, Ind. Eng. Chem., 40, 345 (1948); https://doi.org/10.1021/ie50458a036.

23. S. Kumar, A. Maken, S. Agarwal and S. Maken, J. Mol. Liq., 155, 115 (2010); https://doi.org/10.1016/j.molliq.2010.05.023.
24. A. Maken and S. Maken, J. Ind. Eng. Chem., 18, 1013 (2012); https://doi.org/10.1016/j.jiec.2011.11.139.

25. P.J. Flory, J. Am. Chem. Soc., 87, 1833 (1965); https://doi.org/10.1021/ja01087a002.

26. A. Abe and P.J. Flory, J. Am. Chem. Soc., 87, 1838 (1965); https://doi.org/10.1021/ja01087a003.

27. I. Prigogine, N. Trappeniers and V. Mathot, Discuss. Faraday Soc., 15, 93 (1953); https://doi.org/10.1039/df9531500093.

28. D. Patterson and G. Delmas, Discuss. Faraday Soc., 49, 98 (1970); https://doi.org/10.1039/df9704900098.

29. I. Prigogine, N. Trappeniers and V. Mathot, J. Chem. Phys., 21, 559 (1953); https://doi.org/10.1063/1.1698950.

30. I. Prigogine, A. Bellemans and V. Mathot, The Molecular Theory of Solutions, North-Holland Publishing Company, Amsterdam, edn. 1 (1957).

31. A.J. Treszczanowicz and G.C. Benson, Fluid Phase Equilib., 23, 117 (1985); https://doi.org/10.1016/0378-3812(85)90001-9.

32. M. Rani, S. Agarwal, P. Lahot and S. Maken, J. Ind. Eng. Chem., 19, 1715 (2013); https://doi.org/10.1016/j.jiec.2013.02.011.

33. S. Gahlyan, S. Verma, M. Rani, S. Maken, J. Mol. Liq., 244, 233 (2017); https://doi.org/10.1016/j.molliq.2017.09.015. 